\title{
Osteoporosis treatment: complexities and challenges
}

\author{
R. A. Adler ${ }^{1,2}$
}

Received: 19 January 2016 / Accepted: 24 January 2016 / Published online: 25 February 2016

(C) Italian Society of Endocrinology (SIE) outside the USA 2016

The analogy of cardiac events (e.g., myocardial infarction) and bone events (fractures) can inform our understanding of long-term management of osteoporosis. After a cardiac event, there is no hesitation in applying risk reduction actions, both pharmacologic and non-pharmacologic, whereas it is much less likely that a patient suffering a bone event will have any evaluation and treatment of underlying osteoporosis. This is compounded by the fact that osteoporosis is clinically silent before the fracture and after the fracture has healed, and by the fact that concerns about unusual side effects such as osteonecrosis of the jaw and atypical femoral fractures have led to fewer patients taking osteoporosis medications, particularly anti-resorptive agents [1]. The long-term impact of these phenomena is that while hip fracture incidence has decreased in postmenopausal women in developed countries, there will likely be a plateau followed by an increased incidence of fracture due to the aging of the population, the decrease in use of osteoporosis medications, and the rise of middle class populations in developing countries-people who will now live long enough to fracture.

With potential new agents under development, management of osteoporosis by experts will probably change to a sequence of therapy, perhaps alternating anabolic and anti-resorptive medications. However, most patients with osteoporosis do not see osteoporosis experts, but rather are

R. A. Adler

robert.adler@va.gov

1 Endocrinology and Metabolism, McGuire Veterans Affairs Medical Center, 1201 Broad Rock, Boulevard, Richmond, VA 23249, USA

2 Endocrine Division, Virginia Commonwealth University School of Medicine, Richmond, VA 23298-0111, USA cared for by primary care clinicians and by orthopedic surgeons after a fracture. If the long-term care of osteoporosis becomes complicated and probably expensive, it is likely that some clinicians will either refer the patient to osteoporosis experts (in endocrinology, rheumatology, geriatrics, or internal medicine) or the osteoporosis treatment will be lost because of more pressing, symptomatic disorders of older women and men. Guidelines from medical organizations may help, but the busy clinician is unlikely to read through the many pages of typical guidelines.

There is no "magic bullet" for the management of osteoporosis and prevention of fracture. Current therapy decreases fracture risk by about half. Some patients opt to avoid therapy when they are told that their 10-year hip fracture risk is $5 \%$. A typical response is, "my chance of not having a hip fracture in the next 10 years is $95 \%$ and I am worried about those side effects." Thus, the clinician has to convince the patient that the risk of a fracture greatly outweighs the risk of a side effect. For atypical fractures of the femur, the ratio of typical fractures prevented to atypical fractures associated with anti-resorptive agents is not clear and likely changes substantially with duration of therapy [2]. We are most comfortable in the first 5 years of bisphosphonate treatment, after which it is less established that we are doing no harm. At 5 years, a patient remaining at high risk for fracture might be switched to teriparatide for the next 2 years, but then the patient may need an anti-resorptive again. Such sequential therapy is complicated by the fact that in certain countries, use of teriparatide is severely restricted because of its cost. It is hoped that the introduction of newer anabolic treatments or perhaps shorter treatment regimens will lower the cost of teriparatide.

We hope that odanacatib, abaloparatide, romosozumab, and blosozumab will be safe, effective, and approved therapies for osteoporosis. It might then be possible to tailor 
a sequential program of anti-resorptives, anabolics, and drug holidays to minimize both fracture risk and side effect risks. New surrogates for fracture would bring new drugs to market sooner and may also help the clinician decide the best drug for a specific patient [3]. If it becomes possible to determine which patients are at highest risk for osteonecrosis of the jaw or atypical femoral fracture, anti-resorptive drug choices may be made on a more personalized basis. All of the above applies to the woman with typical postmenopausal osteoporosis or the elderly man with osteoporosis. What about the patient with glucocorticoid-induced osteoporosis or the patient with chronic kidney diseasemetabolic bone disease? Such patients will need even more careful choices of long-term therapy.

It is obvious that while the introduction of nitrogencontaining bisphosphonates started the modern era of osteoporosis treatment, there is still much to do to optimize therapy. Clinicians need new tools to assess patients at baseline and after treatment. Management of this chronic disorder will take knowledge, patience, careful assessment, and patient willingness to use medications portrayed in the media as dangerous, all of this for a silent disorder until there is a fracture. There are not enough "boneheads" to manage all of the patients at risk for fracture. Therefore, we must educate not only our own patients, but also the other clinicians who will evaluate and treat most patients at risk for fracture. This is a daunting task.

\section{Compliance with ethical standards}

Conflict of interest The author has received compensation from Amgen for research consultation on postmenopausal osteoporosis and glucocorticoid-induced osteoporosis.

Ethical approval This article does not contain any studies with human participants or animals performed by the author.

Informed consent Formal consent is not required.

\section{References}

1. Siris ES, Yu J, Bognar K, DeKoven M, Shrestha A, Romley JA, Modi A (2015) Undertreatment of osteoporosis and the role of gastrointestinal events among elderly osteoporotic women with Medicare Part D drug coverage. Clin Interv Aging 10:1813-1824

2. Adler RA, El Hajj Fuleihan G, Bauer DC, Camacho PM, Clarke BL, Clines GA, Compston JE, Drake MT, Edwards BJ, Favus MJ, Greenspan SL, McKinney R Jr, Pignolo RJ, Sellmeyer DE (2016) Managing osteoporosis in patients on long-term bisphosphonate treatment: report of a task force of the American Society for Bone and Mineral Research. J Bone Miner Res 31:16-35

3. FDA Osteoporosis Drug Development Workshop November 4, 2015. www.fda.gov/downloads/drugs/newsevents/ucm472349. pdf, accessed January 18, 2016 